\title{
Planejamento de um Arcabouço Avaliativo para a Identificação do Perfil Evasivo do Discente: Estudo de Caso IFPB Virtual
}

\author{
Alana M. de Morais ${ }^{1}$ \\ ${ }^{1}$ Departamento de Informática - Universidade Federal de Campina Grande (UFCG) - \\ Campina Grande - PB - Brasil \\ alanamorais@copin.ufcg.edu.br
}

\begin{abstract}
The current article designed and proposed a framework for supporting the teacher on the tracking of student dropout in online courses. For this reason, this study mapped metrics related to the student behavioral and adopted a fuzzy architecture to deal with an uncertainty associated with that phenomenon. This framework was built according to the online courses of IFPB Virtual. As main results, we can highlight the proposal framework based on our case study scenario.
\end{abstract}

Resumo. $O$ presente artigo apresenta um arcabouço avaliativo para apoiar o docente no combate à evasão discente em cursos online. Para isto, este estudo fez um mapeamento das métricas comportamentais do aluno relevantes no mapeamento da evasão e adotou uma arquitetura fuzzy para lidar com a incerteza associada a este fenômeno. $O$ arcabouço apresentado foi definido de acordo com a arquitetura dos cursos online do IFPB Virtual. Como resultados iniciais pode-se destacar a proposição de um arcabouço multidimensional de acordo com o cenário do estudo de caso.

\section{Introdução}

Além da democratização do ensino, a Educação a Distância (EaD) apresentou notáveis vantagens sob o ponto de vista da eficiência, principalmente quando há um grande volume de alunos ou se observa o crescimento vertiginoso da demanda por matrículas. A expansão do acesso à $\mathrm{EaD}$ não deve estar relacionada apenas ao aumento do número de vagas em cursos online, mas ao acompanhamento adequado do aprendizado discente e ao apoio dos processos decisórios do professor durante o curso.

Ignorar as especificidades dos sistemas de $\mathrm{EaD}$ ou planejar inadequadamente os cursos online pode agravar problemas pedagógicos e acelerar o insucesso nestas plataformas, identificados na literatura sob o formato de reprovação e evasão. Neste caso, a evasão é um dos problemas mais comuns que afligem as instituições de $\mathrm{EaD}$, pois afetam os resultados dos sistemas educacionais. Assim, a busca por suas causas foi objetivo de muitas pesquisas e investigações (De-La-Fuente-Valentín, Pardo e Kloos, 2013; Rostaminezhad et al., 2013).

A literatura considera como evasão a desistência do curso pelo estudante, independentemente da quantidade de participações efetuadas, bem como as situações de desistência definitiva após determinado contato com o curso. Em outras situações se 
diferencia a evasão ou a permanência no curso de acordo com períodos médios para conclusão de curso e períodos anuais. É sob esse enfoque que o presente artigo seguiu a definição de evasão como a desistência dos discentes de um curso online, ou seja, quando o aluno não completa cursos ou programas de estudo virtuais (Halawa, Greene e Mitchell, 2014). Tais alunos foram identificados como evadidos quando se encontraram sob a seguinte condição: abstenção discente superior a um período de um quarto do tempo de execução do curso online.

A fim de esclarecer o método científico utilizado e os resultados do estudo o presente artigo foi organizado em 5 seções principais. Na segunda seção, discutiu-se trabalhos que almejaram definir o perfil evasivo do discente na literatura. Na terceira seção deste artigo, o planejamento e execução do nosso estudo de caso foram detalhados e discutidos. Além disto, a quarta seção contemplou os principais resultados das investigações. Por fim, a quinta e última seção apresentou as considerações finais e os trabalhos futuros relacionados ao estudo.

\section{Evasão Discente em Cursos Online}

No Brasil, a evasão foi apontada por grande parte das instituições como um dos maiores obstáculos enfrentados nos diferentes tipos de cursos online. De acordo com o Censo Ead.BR, as instituições investigadas apontaram taxas de evasão em torno de $40 \% \mathrm{em}$ todos os tipos de cursos online no Brasil em 2015 (Censo EaD.BR, 2016). Contudo, a problemática da evasão discente não se limitou ao contexto brasileiro, pois diversas instituições internacionais relataram índices tão alarmantes quando os nacionais. Por exemplo, a Universidade Duke ofertou um curso de Bioeletricidade em 2012, por meio do ambiente Coursera, e obteve 12.175 estudantes inscritos. No entanto, apenas $63,74 \%$ dos estudantes assistiram a um vídeo, 30,04\% interagiram com um questionário, 2,83\% tentaram o exame final e, finalmente, apenas 2,57\% obtiveram o certificado (Yang et al., 2013). Relata-se que a taxa de desistência em geral atingiu entre $91 \%$ a $93 \%$ nestes ambientes virtuais.

A evasão pode ocorrer por uma série de razões, mas a maioria das pesquisas focou em elencar fatores pessoais, profissionais e educacionais (Costa, 2013). Por exemplo, o estudo de Lee e Choi (2011) observou que as razões para a evasão puderam ser divididas, de modo geral, em fatores motivacionais internos (que influenciaram o desejo de um aluno a persistir no curso) e fatores externos. O Censo EaD.BR (2016), por exemplo, afirmou que as instituições apontaram o fator tempo como o mais influente diante do fenômeno da evasão, seguido pelo fator finanças. Neste mesmo sentido, há a pesquisa de Ribeiro et al. (2014) que identificou parâmetros que contribuíram para minimizar os índices de desistência por meio de análises dos relatórios de conclusão e da correlação destes com a avaliação da percepção dos alunos ao final de duas edições do curso. Para isto, os autores se propuseram a avaliar 8 fatores, extraídos por meio de um questionário qualitativo, que permearam a qualidade de cursos em EaD: aprendizagem efetiva, entusiasmo, organização do tutor online, interação com o tutor, relação com o tutor, abrangência do conteúdo, processos avaliativos e atividades. Os dados produziram um fator de risco de evasão, o qual foi constantemente monitorado e avaliado pelos gestores do curso ao final de cada semestre.

Por outro lado, há pesquisas que identificaram fatores comportamentais do discente relacionadas ao fenômeno da evasão nos cursos online. Este é o principal aspecto investigado pelo presente artigo, pois são estas variáveis que descrevem o 
comportamento do aluno no ambiente virtual. Além disto, tais medidas podem ser ajustadas ao longo do processo de ensino-aprendizagem por meio de mensagens feedback e de novas atividades pedagógicas pelo docente de acordo com seus objetivos.

Como destaque inicial, há o estudo de Mezzari et al. (2013) que realizou um apanhado sobre os aspectos relacionados ao fenômeno da evasão na $\mathrm{EaD}$. Os autores defenderam a participação dos alunos nos ambientes virtuais como indicativo eficiente na detecção de alunos em perigo de evasão. Inicialmente, o estudo buscou validar alguns indicadores de desempenho, propostos por Araújo e Lucena (2005), são eles: tipo de participação, tipo de interação e tipo de comunicação. Neste sentido, tais índices permitiram a avaliação das interações e correlação dos resultados obtidos com o desempenho da equipe. Por fim, o trabalho destacou como estratégia para detecção da evasão do discente um processo de acompanhamento contínuo e permanente deste durante o curso.

Outro trabalho relevante para a presente análise foi a pesquisa de Rostaminezhad (2013), que tencionou encontrar fatores relacionados ao complexo fenômeno da evasão em cursos online. Este estudo identificou uma série de critérios que afetaram direta ou indiretamente os alunos. Neste sentido, três critérios se destacaram de acordo com a literatura pesquisada: a satisfação do aluno como um fator de motivação, autorregulação e interação. Além disto, foi executado um estudo de caso com estudantes para investigar a importância de tais métricas. A amostra coletada foi composta por 877 alunos de EaD da Universidade de Ciência e Tecnologia do Irã. Inicialmente, o estudo de caso sobre os discentes iranianos revelou a existência de uma correlação forte entre a autorregulação e o sucesso do aluno. Neste mesmo contexto, em relação à satisfação e interação dos discentes mais pesquisas devem ser necessárias, pois as correlações se mostraram fracas.

Assim, limitar e minimizar o abandono é um dos principais objetivos e desafios de instituições que oferecem cursos nesta modalidade. Por isto, o primeiro passo para uma contribuição efetiva para este desafio é identificar quais métricas do discente, relacionadas à interação do aluno com o ambiente virtual, podem estar relacionadas a sua desistência no curso. Este artigo tem como intuito propor soluções para ambientes virtuais que possuam características semelhantes aos cursos online providos pela Universidade Aberta do Brasil (UAB). Neste sentido, um estudo preliminar voltado a este cenário e a definição de métricas comportamentais relevante já foi publicado anteriormente (Morais et al., 2014).

\section{Métodos}

Esta seção tem como intuito apresentar e discutir o método científico utilizado (estudo de caso) e o cenário selecionado na proposição do arcabouço avaliativo.

\subsection{Cenário selecionado: IFPB Virtual}

O IFPB Virtual é composto por três grupos principais de cursos, são eles: cursos superiores (Letras, Administração e Licenciatura em Computação), cursos técnicos (Segurança no trabalho e Secretaria Escolar) e cursos de pós-graduações (Gestão Pública). As análises executadas foram realizadas com os dados armazenados no ambiente virtual Moodle e contempla registros de tais cursos entre os períodos 2012.2 a 
VI Congresso Brasileiro de Informática na Educação (CBIE 2017)

Anais do XXIII Workshop de Informática na Escola (WIE 2017)

2015.2. Todos os cursos analisados foram avaliados por meio da execução de um estudo de caso das interações dos cursos já encerrados.

O ambiente virtual Moodle (http://www.ifpb.edu.br/ead) possui aproximadamente mais de 300 tabelas em seu banco de dados e, para extrair determinada informação, foi feito um mapeamento das tabelas que ali estão. Assim, ocorreu um processo de análise e refinamento para obter apenas informações relevantes aos propósitos do estudo. Entre elas é possível destacar três grupos principais de tabelas relacionadas aos usuários, cursos e atividades realizadas pelo usuário foram analisados durante o presente estudo, além da tabela de registro das interações $(\log )$.

\subsection{Estudo de caso}

Este estudo executou uma pesquisa de campo do tipo estudo de caso descritivo (Yin, 2005), no qual foram traçados e identificados os fatores que influenciaram os alunos a evadir dos cursos na plataforma do IFPB Virtual, com base nos critérios motivação, autorregulação e interação como destacado no trabalho de Rostaminezhad (2013).

Mediante a tais critérios, foram selecionados diversos fatores (variáveis) para serem candidatas a participar ou não do arcabouço que este trabalho almejou construir neste estudo. As métricas eram selecionadas por dois critérios principais no presente estudo: ser relevante para descrever um dos três critérios selecionados e ter correlação forte com o fenômeno evasão no curso em análise. Todas as variáveis analisadas possuíam um caráter quantitativo.

Neste momento do artigo, é interessante entender o que significa cada um destes critérios frente as teorias da Psicologia Cognitiva e da Pedagogia, para facilitar o processo de entendimento e a extração de tais informações na base de dados analisada.

\subsubsection{Autorregulação}

A aprendizagem pode ser definida como autorregulada quando o aluno se sente livre para influenciar sobre o que, quando, onde e como aprender (Weinert, 1982). Neste sentido, alunos autorreguladores da sua aprendizagem desenvolvem a consciência do que podem ou não realizar, do que entendem e do que não entendem de modo a encontrar recursos para atingir os seus objetivos definidos (Rosário et al., 2010). No sentido de realizar uma tarefa com sucesso, procuram a informação que precisam, percorrendo os passos necessários para a sua execução, mesmo quando encontram obstáculos (Zimmerman, 2008). Os alunos autorregulados, na sua aprendizagem, estabelecem objetivos realistas, e recorrem a uma bateria de recursos, abordando as tarefas acadêmicas com confiança. A aprendizagem autorregulada é vista como um mecanismo para ajudar a explicar as diferenças de desempenho entre os alunos e como melhorar a sua resolução de problemas.

\subsubsection{Motivação}

Diversas pesquisas evidenciaram que a motivação pode produzir um efeito na aprendizagem e no desempenho dos estudantes. Os princípios da teoria da Autodeterminação apontam que as motivações dos indivíduos diferem e são determinadas pelos contextos que dão subsídios a necessidades psicológicas com diferentes manifestações (Leal et al., 2013), o que torna a motivação dos estudantes para 
a aprendizagem "um fenômeno complexo, multideterminado, que pode apenas ser inferido mediante a observação do comportamento" (Guimarães e Bzuneck, 2008, p.111). De acordo com a teoria da Autodeterminação, a motivação de um indivíduo pode ser classificada em três tipos principais: desmotivação, motivação extrínseca e motivação intrínseca. A desmotivação, como o próprio nome diz, engloba indivíduos que apresentam ausência de motivação, ou seja, o aluno não apresenta intenção nem comportamento proativo e "em tal situação, observa-se desvalorização da atividade e falta de percepção de controle pessoal” (Guimarães e Bzuneck, 2008, p. 103).

\subsubsection{Interação}

A análise das interações aborda uma série de relações entre discentes, docentes e conteúdo nos ambientes virtuais, como: relacionamento aluno-professor, entre alunos e aluno-conteúdo (Fuks e Pimentel, 2011). O outro tipo de interação que precisa ser comentada é a relação aluno-conteúdo, pois os cursos online permitem o desenvolvimento dos conteúdos e objetos de aprendizagem de diversas formas: som, texto, imagens, vídeo e realidade virtual. Além disso, o discente pode interagir com o conteúdo de diversas maneiras: explorando, selecionando, controlando, construindo, respondendo, entre outras maneiras. Por fim, o último tipo de interação que esta seção destaca se refere ao relacionamento entre alunos.

\section{Resultados: FuzzySD}

O trabalho propôs um arcabouço metodológico a fim de otimizar as análises, sejam elas formativas ou somativas, baseando-se em uma análise multidimensional dos discentes nos cursos online. $\mathrm{O}$ arcabouço avaliativo (chamado de FuzzySD) teve como intuito analisar os indicadores associados às atividades do aluno no ambiente e, com isso, prover contribuições à avaliação dos discentes por meio do monitoramento de métricas que descrevam suas interações nos cursos online.

De acordo com revisão de literatura executada no estudo, algumas métricas de comportamento do discente puderam ser incorporadas como entradas do arcabouço planejado, pois tais critérios apresentaram relevância durante as análises. Diante destas métricas, três grupos merecem destaque: métricas relacionadas à Autorregulação do aluno, métricas relacionadas à Interação do aluno, e métricas relacionadas à Motivação do aluno.

Tabela 1. Critérios e as variáveis associadas a estes no estudo de caso (antes da análise de correlações)

\begin{tabular}{|c|c|c|}
\hline Autorregulação & Interação & Motivação \\
\hline Média das Notas & $\begin{array}{c}\text { Número de } \\
\text { visualizações }\end{array}$ & $\begin{array}{c}\text { Polaridade das mensagens de } \\
\text { fórum (Mensagens dos fóruns) }\end{array}$ \\
\hline $\begin{array}{c}\text { Interação com o } \\
\text { professor }\end{array}$ & $\begin{array}{c}\text { Interação com outros } \\
\text { alunos (bate-papo e } \\
\text { fóruns de discussão) }\end{array}$ & $\begin{array}{c}\text { Polaridade das mensagens de } \\
\text { chat (Mensagens de chat) }\end{array}$ \\
\hline
\end{tabular}


VI Congresso Brasileiro de Informática na Educação (CBIE 2017)

Anais do XXIII Workshop de Informática na Escola (WIE 2017)

\begin{tabular}{|c|c|c|}
\hline Edição de recursos & $\begin{array}{c}\text { Dias sem acessar o } \\
\text { sistema }\end{array}$ & \\
\hline
\end{tabular}

Após a seleção das variáveis relacionadas aos critérios, foram executadas análises de correlação em uma amostra de treinamento proveniente de dados de cursos do IFPB Virtual. Foram executadas diversas rodadas de análises de correlação, pois foram envolvidos cursos de diferentes características (superiores, técnicos e de pós-graduação). Porém, a título de informação este artigo apresentou os resultados referentes ao primeiro período do curso de Letras. A Figura 1 ilustrou os resultados da análise de correlação de Spearman executada diante de uma das amostras de treinamento normalizadas. É possível perceber que apenas a variável bate-papo (que se refere a quantidade de interações via bate-papo) possui uma correlação fraca de 0,2 . Tal comportamento se repetiu em outras rodadas e nos fez perceber que no cenário selecionado as interações de bate-papo são pouco influentes na evasão do discente no curso. Por isso, umas das decisões tomadas foi a exclusão das variáveis de entrada bate-papo dos critérios.

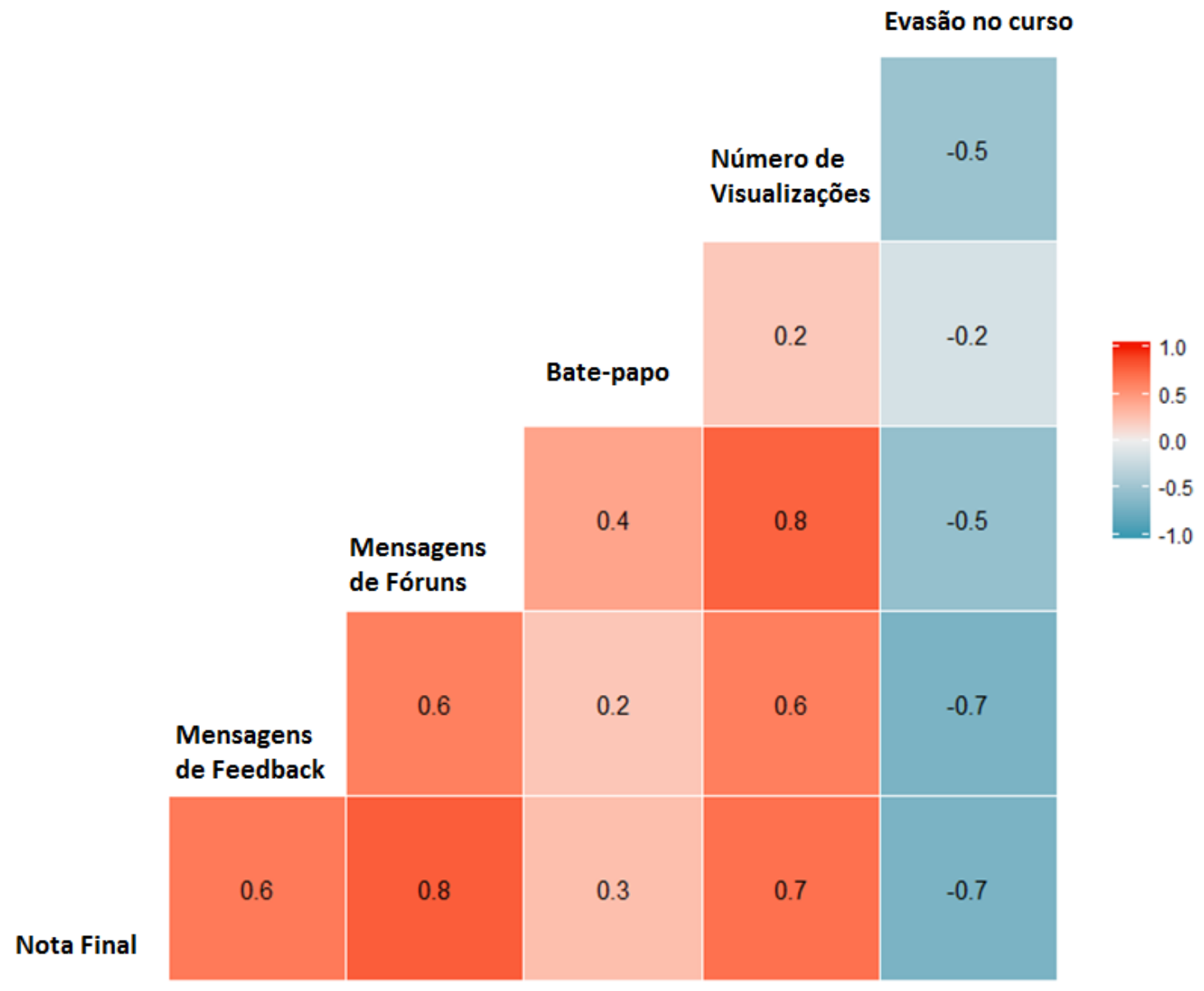

Figura 1. Análises de correlação

A Tabela 2 ilustra como ficaram as entradas após as análises de correlação entre as variáveis e a evasão do aluno. Todas os grupos de métricas possuem um caráter fuzzy definidos pelo especialista da educação no cenário investigado. Por isso, o arcabouço foi estruturado diante das técnicas de inferência fuzzy (Figura 2). 
VI Congresso Brasileiro de Informática na Educação (CBIE 2017)

Anais do XXIII Workshop de Informática na Escola (WIE 2017)

Tabela 2. Critérios e as variáveis associadas a estes no estudo de caso (após análise de correlações)

\begin{tabular}{|c|c|c|}
\hline Autorregulação & Interação & Motivação \\
\hline Média das Notas & $\begin{array}{c}\text { Número de } \\
\text { visualizações }\end{array}$ & $\begin{array}{c}\text { Polaridade das mensagens de } \\
\text { fórum (Mensagens dos fóruns) }\end{array}$ \\
\hline $\begin{array}{c}\text { Interação com o } \\
\text { professor }\end{array}$ & $\begin{array}{c}\text { Interação com outros } \\
\text { alunos (fóruns de } \\
\text { discussão) }\end{array}$ & \\
\hline Edição de recursos & Dias sem acessar o & \\
& sistema & \\
\hline
\end{tabular}

$\mathrm{O}$ arcabouço delineado deixou em aberto a possibilidade de inserção de novas métricas por meio do critério "Outros Fatores". Tal estratégia foi adotada por identificar algumas especificidades de acordo com o cenário utilizado na aplicação do arcabouço. A proposta tencionou adaptar e validar a abordagem avaliativa a diferentes contextos de cursos online, seguindo o pré-requisito de seguir a estrutura de ensino definida pela UAB.

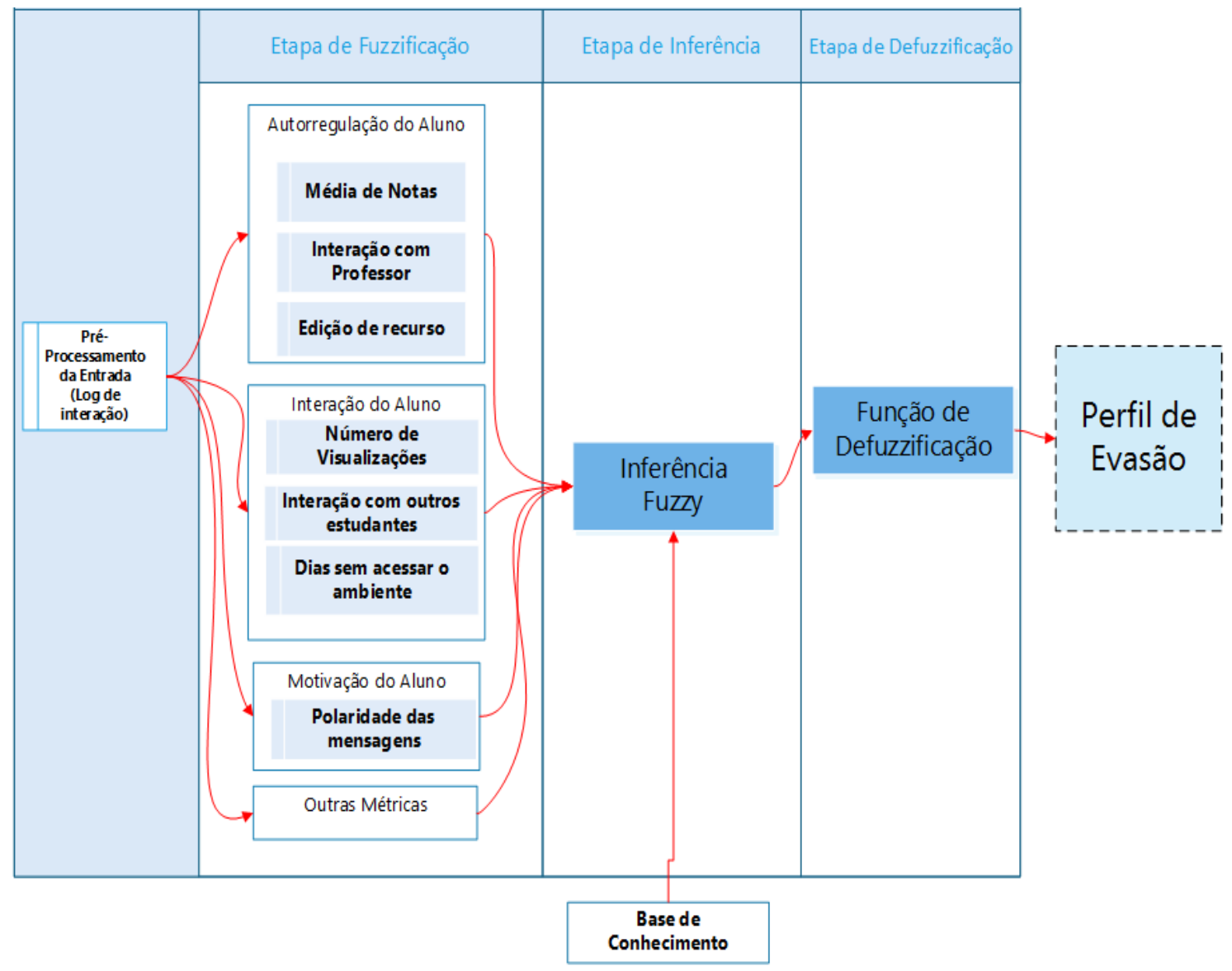

Figura 2. Arcabouço Proposto - FuzzySD 
VI Congresso Brasileiro de Informática na Educação (CBIE 2017)

Anais do XXIII Workshop de Informática na Escola (WIE 2017)

\section{Conclusões}

O presente trabalho possui como principal objetivo a proposição de um arcabouço avaliativo com o intuito de combater uma das problemáticas mais persistentes na EaD: a evasão. Os prejuízos econômicos, sociais e pessoais causados pela evasão mostram como é urgente estudos e medidas que visem combater tal fenômeno. Este artigo se propôs a apresentar o processo de planejamento e metodologia para a construção de um arcabouço avaliativo fuzzy.

No tocante às ameaças de validade do sistema, inicialmente já se pode destacar a limitação de ser focado em um tipo de sistema de $\mathrm{EaD}$, neste caso estruturas semelhante aos cursos online da UAB. O arcabouço tentou minimizar tal ameaça adicionando uma entrada "outros fatores", mas ainda é necessário avaliar o arcabouço em outro cenário de EaD.

O presente arcabouço está na fase de validação e automatização do processo de geração de regras fuzzy. A principal contribuição deste estudo é propor uma alternativa para a minimização dos índices de evasão. Além disso, tem-se como contribuição computacional a modelagem fuzzy do cenário e uma alternativa para a predição de comportamentos do discente em cursos online.

Como trabalhos futuros é importante destacar a finalização da validação do arcabouço por meio de uma análise temporal.

\section{Agradecimentos}

Gostaria de agradecer ao IFPB Virtual pela parceria e por apoiar a pesquisa na área de Informática na Educação.

\section{Referências}

Bezerra, C., Scholz, R., Adeodato, P., Lucas, T. and Ataide, I. (2016) Evasão Escolar: Aplicando Mineração de Dados para Identificar Variáveis Relevantes. In Anais do Simpósio Brasileiro de Informática na Educação-SBIE. Vol. 27, No. 1, p. 1096.

Rostaminezhad, M. A., Mozayani, N., Norozi, D., Iziy, M. (2013) Factors Related to Elearner Dropout: Case Study of IUST Elearning Center. Procedia-Social and Behavioral Sciences, Vol. 83, p. 522-527.

De-La-Fuente-Valentín, L.; Pardo, A.; Kloos, C. D. (2013) Addressing drop-out and sustained effort issues with large practical groups using an automated delivery and assessment system. In Computers \& Education. vol. 61, p. 33-42.

Halawa, S.; Greene, D.; Mitchell, J. (2014) Dropout Prediction in MOOCs using Learner Activity Features. In: Proceedings of European MOOCs Stakeholders Summit (EMOOCs). p. 58-65.

Morais, A. M.; Araujo, J.M.F.R.; Costa, E. B. (2014) Arcabouço Multidimensional de Avaliação dos Discentes baseado na Análise de Recursos Pedagógicos. XXII Workshop sobre Educação em Informática-2014-Brasília, DF. 
VI Congresso Brasileiro de Informática na Educação (CBIE 2017)

Anais do XXIII Workshop de Informática na Escola (WIE 2017)

Ribeiro, G.O.; Silva, T.E.; Nunes, A.O.; Pinto, F.A.P.; Vasconcelos, F.H.L. (2014) Perspectivas para a Redução da Evasão em EaD a partir da Avaliação da Qualidade do Ensino Online. In Anais do Workshop de Informática na Escola (Vol. 20, No. 1, p. 428).

Araujo, L.; Lucena, G. (2005) Comunidades virtuais de aprendizagem: novas dinâmicas de aprendizagem exigem novas formas de avaliação. In: XVI Simpósio Brasileiro de Informática na Educação. SBC/ UFJF, Anais ... Juiz de Fora, MG. 2005.

Censo EAD.BR: Relatório Analítico da Aprendizagem a Distância no Brasil $2015=$ Censo EAD.BR: Analytic Report of Distance Learning in Brazil 2015/(organização) ABED - Associação Brasileira de Educação a Distância; (traduzido por Maria Thereza Moss de Abreu). Curitiba: InterSaberes, 2016.

Costa, R. (2013) Educação a distância: um desafio atual. Educação a Distância: elementos para pensar o ensino-aprendizagem contemporâneo. Daniel Mill, Cristiano Maciel (Org.). Cuiabá: EdUFMT, 2013. 369p

Fuks, H.; Pimentel, M. (2011). Sistemas colaborativos. Elsevier Brasil.

Guimarães, S. E. R.; Bzuneck, J. A. (2008). Propriedades psicométricas de um instrumento para avaliação da motivação de universitários. Ciências \& Cognição, Ilha do Fundão, 13 (1), 101-113.

Leal, E.A.; Miranda, G.J.; Carmo, C.R.S. (2013) Teoria da autodeterminação: uma análise da motivação dos estudantes do curso de ciências contábeis. Revista Contabilidade \& Finanças-USP, 24(62). 2013

Lee, Y.; Choi, J. (2011) A review of online course dropout research: implications for practice and future research. Educational Technology Research and Development, v. 59, n. 5, pp. 593-618. 2011.

Mezzari, A.; Rockenbach, L.; Gorziza, B.; Ribas, G.; Favero, R.; Bulegon, A. (2013) Estratégias para detecção precoce de propensão à evasão. RIED, v. 16, n. 2, pp 147175. 2013. ISSN: 1138-2783.

Rosário, P., Núñez, J. C., Valle, A., González-Pienda, J., Lourenço, A. (2013). Grade level, study time, and grade retention and their effects on motivation, self-regulated learning strategies, and mathematics achievement: a structural equation model. European Journal of Psychology of Education, 28(4), 1311-1331.

Weinert, F. E. (1982). Selbstgesteuertes Lernen als Voraussetzung, Methode und Ziel des Unterrichts [Self-regulated learning as prerequisite, method and objective of instruction], Unterrichtswissenschaft, 10 (2), pp. 99-110.

Yang, D.; Sinha, T.; Adamson, D.; Rosé, C. P. (2013) Turn on, tune in, drop out: Anticipating student dropouts in massive open online courses. In: Proceedings of the 2013 NIPS Data-Driven Education Workshop. Anais Nevada, USA. 2013. p. 1-8.

Yin, R. K. (2005) Estudo de caso: planejamento e métodos. $3^{\text {a }}$ ed. Porto Alegre: Bookman, 2005. $212 \mathrm{p}$. 
VI Congresso Brasileiro de Informática na Educação (CBIE 2017)

Anais do XXIII Workshop de Informática na Escola (WIE 2017)

Zimmerman, B. J. (2008) Investigating self-regulation and motivation: historical background, methodological developments, and future prospects. American Educational Research Journal, 45(1), 166-183. 2008. 